\title{
An evolutionary approach for the motion planning of redundant and hyper-redundant manipulators
}

\author{
Maria da Graça Marcos - J.A. Tenreiro Machado • \\ T.-P. Azevedo-Perdicoúlis
}

\begin{abstract}
The trajectory planning of redundant robots is an important area of research and efficient opti- mization algorithms are needed. The pseudoinverse control is not repeatable, causing drift in joint space which is undesirable for physical control. This paper presents a new technique that combines the closed- loop pseudoinverse method with genetic algorithms, leading to an optimization criterion for repeatable con- trol of redundant manipulators, and avoiding the joint angle drift problem. Computer simulations performed based on redundant and hyper-redundant planar manipulators show that, when the end-effector traces a closed path in the workspace, the robot returns to its initial configuration. The solution is repeatable for a workspace with and without obstacles in the sense that, after executing several cycles, the initial and fi- nal states of the manipulator are very close.
\end{abstract}

Keywords Redundant manipulators, Hyper-redundant, manipulators, Robots, Kinematics, Genetic algorithms, Trajectory planning

\section{Introduction}

Kinematic redundancy occurs when a manipulator possesses more degrees of freedom than the required to execute a given task. In this case the inverse kine- matics admits an infinite number of solutions, and a criterion to select one of them is required. Most of the research on redundancy deals with the use of these ex- tra degrees of freedom and is referred to in the litera- ture as the resolution of redundancy [1].
Many techniques for solving the kinematics of redundant manipulators that have been suggested con- trol the end-effector indirectly, through the rates at which the joints are driven, using the pseudoinverse of the Jacobian (see, for instance, [2]). The pseudoin- verse of the Jacobian matrix guarantees an optimal reconstruction of the desired end-effector velocity - in the least-squares sensewith the minimum-norm joint velocity. However, even though the joint veloc- ities are instantaneously minimized, there is no guar- antee that the kinematic singularities are avoided [3]. Moreover, this method has the generally undesirable property that repetitive end-effector motions do not necessarily yield repetitive joint motions. Klein and Huang [4] were the first to observe this phenomenon for the case of the pseudoinverse control of a pla- nar three-link manipulator. A large volume of research 
has been produced in the last few years in this topic [5-8]. For example, Zhang et al. [9] solve the joint angle drift problem by means of a dual-neuralnetwork based quadratic-programming approach.

Baillieul [10] proposed a modified Jacobian matrix called the extended Jacobian matrix. The extended Ja- cobian is a square matrix that contains the additional information necessary to optimize a certain function. The inverse kinematic solutions are obtained through the inverse of the extended Jacobian. The algorithms, based on the computation of the extended Jacobian matrix, have a major advantage over the pseudoinverse techniques, because they are locally cyclic [11]. The disadvantage of this approach is that, while mechan- ical singularities may be avoided, typical algorithmic singularities [12] arise from the way the constraint re- stricts the motion of the mechanism [13].

One optimization method that is gaining popularity for solving complex problems in robotics is the Ge- netic Algorithm (GA). GAs are populationbased sto- chastic and global search methods. Their performance is superior to that revealed by classical optimization techniques [14] and has been used successfully in ro- bot path planning.

Parker et al. [15] used GAs to position the end- effector of a robot at a target location, while min- imizing the largest joint displacement. This method has some shortcomings, such as the lack of precision, and is affected by the values of the weights. Arakawa et al. [16] proposed a virusevolutionary genetic algo- rithm, composed of a host population and a virus pop- ulation with subpopulations, for the trajectory genera- tion of redundant manipulators without collision, that optimize the total energy. The operators of crossover, mutation, virus infection and selection are executed in each subpopulation independently. Kubota et al. [17] studied a hierarchical trajectory planning method for a redundant manipulator using a virus-evolutionary GA. This method runs, simultaneously, two processes. One process calculates some manipulator collision-free po- sitions and the other generates a collision-free trajec- tory by combining these intermediate positions. De la Cueva and Ramos [18] proposed a GA for planning paths without collisions for two robots, both redundant and non-redundant, sharing the same workspace. The GA works directly over the task space adopting 
proposed a motion planning method using an artifi- cial potential field and a GA for a hyperredundant manipulator whose workspace includes several obsta- cles. The motion planning is divided into two sub- problems. The first is the "Path planning" that gener- ates a trajectory leading the tip of manipulator to the goal without collisions, using the artificial potential field concept. The second consists in the "Collision- free sequence generation" that generates a sequence of movements by which distinct parts of the manipulator can avoid collisions with the obstacles. McAvoy and Sangolola [20] proposed an approach with GAs for optimal point-to-point motion planning of kinemat- ically redundant manipulators. Their approach com- bines B-spline curves, for the generation of smooth trajectories, with GAs, for obtaining the optimal so- lution. Peng and Wei [21] presented the ASAGA trajectory planning method of redundant manipulators by combining a stochastic search algorithm (simulated annealing algorithm) and a GA. In the ASAGA the se- lection, crossover and mutation operators are adjusted by using an adaptive mechanism based on the fitness value. the direct kinematics. Each robot is associated with

one population and each string of a population rep-

resents a complete robot path. Nishimura et al. [19]
Zhang et al. [22] proposed an algorithm to solve the inverse kinematics of a flexible macro-micro manipulator system which combines a GA and a neural network. Pires et al. [23] proposed a multi-objective genetic algorithm, when considering up to five simul- taneous objectives, to generate manipulator trajecto- ries and for obstacle avoidance.

Having these ideas in mind, the paper is organized as follows. Section 2 introduces the fundamentals of the kinematics of redundant manipulators. Based on these concepts, Sect. 3 presents the new closed- loop inverse kinematics algorithm with genetic al- gorithms (CLGA) and the open-loop genetic algo- rithm (OLGA). Section 4 presents the simulation re- sults in a workspace without and with obstacles. For comparison purposes some results for the closed-loop pseudoinverse (CLP) method are also presented. Finally, Sect. 5 draws the main conclusions.

\section{Kinematics and dynamics of redundant manipulators}

We consider a manipulator with $n$ degrees of freedom whose joint variables are denoted by $\mathbf{q}=\mathrm{T}$

$[q 1, q 2, \ldots, \quad$. We assume that the class of tasks $\left.q_{n}\right]$ we are interested in Tr an be described by $m$ variables, $\mathbf{x}=[x 1, x 2, \ldots, x m], m<n$, and that the relation be- 
tween $\mathbf{q}$ and $\mathbf{x}$ is given by the direct kinematics:

$x=f(q)$

Differential kinematics of robot manipulators was introduced by Whitney [24] that proposed the use of differential relationships to solve for the joint motion from the Cartesian trajectory of the endeffector. Dif- ferentiating (1) with respect to time yields:

$\dot{x}=J(q) q$

where $\mathbf{x} \in \mathrm{R}^{m}, \boldsymbol{q} \in \mathrm{R}^{n}$ and $\mathrm{J}(\mathbf{q})=\partial f(\mathbf{q}) / \partial q \in \mathrm{R}^{m \times n}$. Hence, it is possible to calculate a path $\mathbf{q}(t)$ in terms

of a prescribed trajectory $\mathbf{x}(t)$ in the operational space.

Equation (2) can be inverted to provide a solution in terms of the joint velocities:

$q=J^{\#}(q) x$

where $\mathbf{J}^{\#}$ is the Moore-Penrose generalized inverse of the Jacobian $\mathbf{J}[2,25]$.

\section{Robot trajectory control}

The Jacobian of an $n$-link planar manipulator (i.e., $m=2$ ) has a simple recursive nature according to the

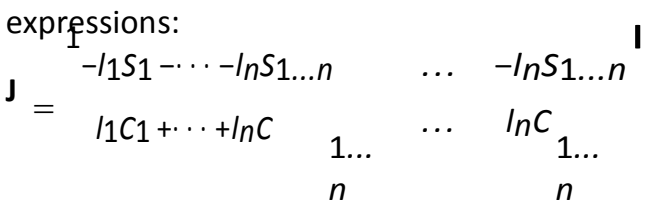

where $l i$ is the length of link $i, q i \ldots k=q i+\cdots+q k$, Si...k $=\operatorname{Sin}(q i \ldots k)$ and $C_{i} \ldots k=\operatorname{Cos}(q i \ldots k), i, k \in N$. the

In the closed-loop pseudoinverse (CLP) method

joint positions can be computed through the time inte- gration of the expression:

$.6 . q=J^{\#}(q) \cdot 6 . x$

where .6. $\mathbf{x}=\mathbf{x}$ ref $-\mathbf{x}$ and $\mathbf{x}$ ref is the vector of reference position in the operational space. Nevertheless, in a previous study, addressing the CLP method [26], it

was concluded that this method leads to unpredictable,

not repeatable arm configurations and reveals proper- ties resembling those that occur in chaotic that maximizes the fitness or the cost function. The GA modifies repeatedly the population of individuals (possible solutions). At each step, the genetic algo- rithm selects individuals at random, from the current population, to be parents, and uses them to produce the offspring for the next generation. Over successive generations, the population evolves towards an opti- mal solution. The GAs can be applied to solve a vari- ety of optimization problems that are not well suited for standard optimization algorithms, including problems in which the objective function is discontinuous, not differentiable, stochastic, or highly nonlinear.

Bearing these facts in mind, in this paper we propose a new method that combines the CLP with a GA, namely the closed-loop inverse kinematics algorithm with genetic algorithms (CLGA).

In order to find an initial joint configuration of the manipulator, another GA is used, adopting the direct kinematics, which is denoted as OLGA.

In both cases the optimal configuration is the one that minimizes the fitness function according to some specified criteria.

\subsection{The CLGA formulation}

The CLGA adopts the closed-loop structure without requiring the calculation of the pseudoinverse. $n \times n$ The CLGA uses an extended Jacobian matrix, $\mathrm{J}^{*} \in \mathrm{R}$, and an extended vector, .6. $\mathrm{x}^{*} \in \mathrm{R}^{n}$, as a way to limit the joint configurations for a given end-effector position.

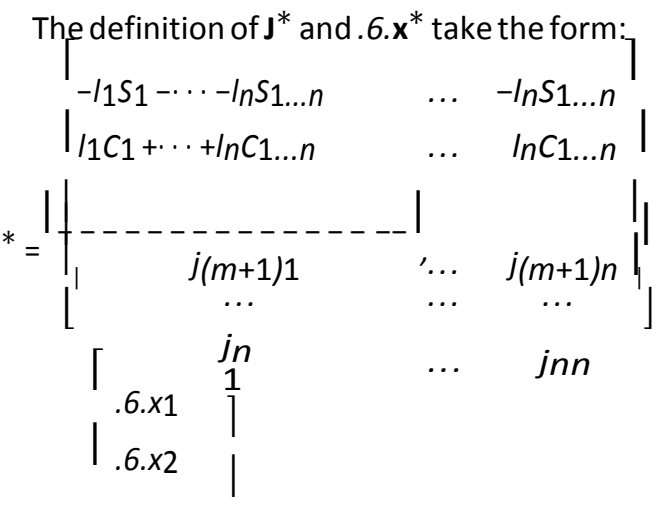




$$
\left|6 . x_{m+1}\right|
$$

Genetic algorithms (GAs) comprise a method for solving both constrained and unconstrained optimiza- tion problems, based on the mechanics of natural ge- netics and selection, first introduced by Holland [27]. A GA allows a population composed of many individ- uals to evolve under specified selection rules to a state
$.6 \cdot x^{*}=\left|\begin{array}{|l}\mid-- \\ \therefore \\ 6 \times n\end{array}\right|$

where the matrix elements $j i k$ and $.6 . x i, i=$ $m+1, \ldots, n$ and $k=1, \ldots, n$, are values generated by the $G A$, satisfying the additional imposed constraints. 


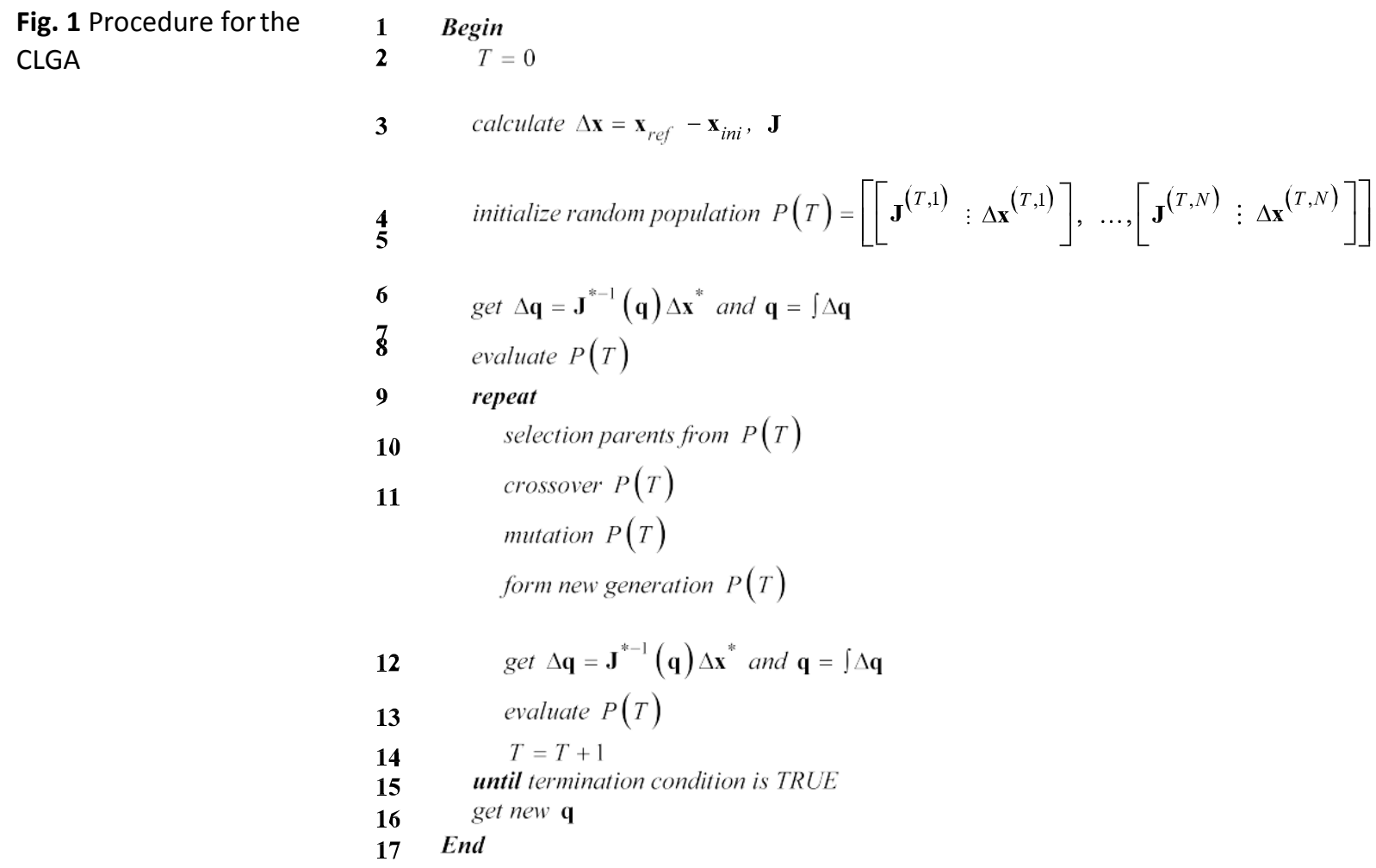

\subsubsection{Representation and operators in the CLGA}

An initial population of strings, with dimension $n P=N$, is constructed at random and the search is then carried out among this population. Each chro- mosome (string) is implemented by a matrix of $n V=(n-m) \times(n+1)$ values (genes), with $j d k$ and .6. $x d, d=1, \ldots, n-m, k=1, \ldots, n$, consisting in floating- point numbers initialized in the range [varmin, varmax]. For the generation $T$, the ith chromosome of the pop-

ulation is represented as:

$\begin{array}{ll}\mathrm{j}_{\mathrm{J}}(T & (T \\ , i) \cdot .6 . \mathrm{x} & , i)^{\mathrm{l}}\end{array}$

where

$\mathrm{J}(T, i)={ }_{j}^{\mathrm{r}}(T, i)^{\mathrm{l}} \in \mathrm{R}(n-m) \times n$

an are randomly grouped into pairs and a crossover point is randomly selected for each one of the $n-m$ lines of the parent. Then crossover is performed among pairs.

Finally, for the mutation operator, one variable value from the $n V$ values of the chromosome is replaced with a new random one.

The CLGA procedure is shown in Fig. 1, where xref is the vector of reference position in the operational space and $\mathbf{x i n i}$ is a vector representing the position of the end-effector in the operational space.

\subsubsection{Optimization criteria}

(7) The fitness function minimizes the joint displacement between the current joint position and the initial joint position, through the following function:

$$
q^{q}{ }^{T} \text { q } q
$$

$$
f 1=A \dot{\mathrm{q}} \mathrm{q}+B \quad \begin{array}{lll}
0 & -0 \\
\text { q. } & . t & .6 . t
\end{array}
$$


gorithm are reproduction, crossover and mutation. In what concerns the reproduction operator, the succes- sive generations of new strings are generated on the basis of their fitness function. In this case, it is used a rank weighting to select the strings from the old to the new population. For the crossover operator, the strings where $A, B \in \mathrm{R}^{+}$denotes weighting factors, $\mathbf{q} 0$ and $q$ represent the initial and current joint configurations, respectively, and .6.t is a step time increment.

\subsection{The OLGA formulation}

The OLGA trajectory planning adopts a simple openloop structure, as we can see in Fig. 2. An initial pop- 


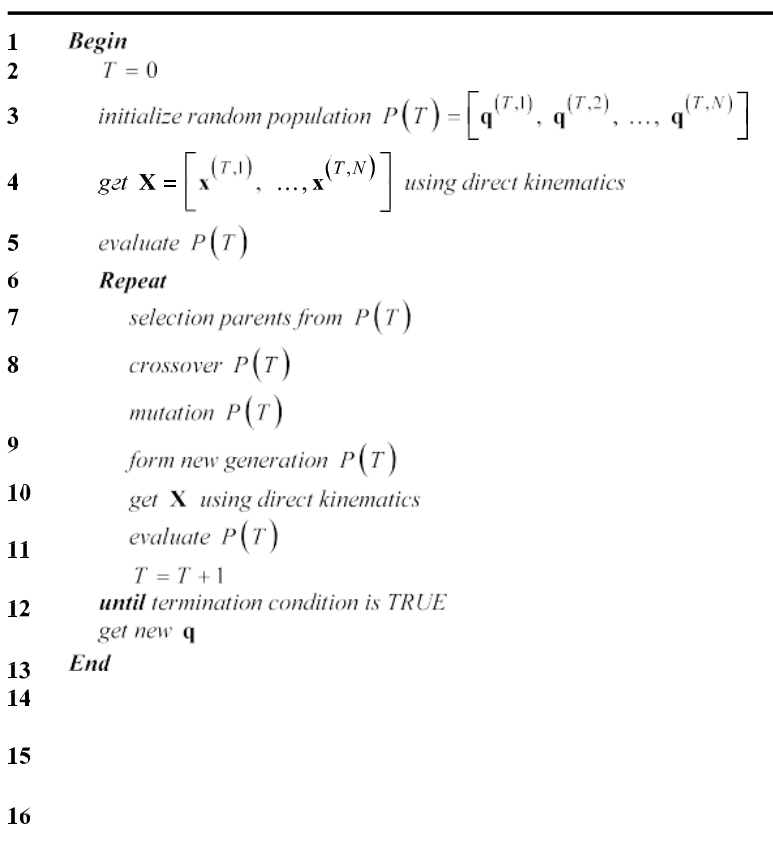

Without lack of generality, in the following experiments are adopted arms having identical link lengths, $11=/ 2=\cdots=\ln$.

The experiments consist in the analysis of the kinematic performance of a planar manipulator with $n=$ $\{3,4,5,6,7\}$ rotational joints, denoted as $n R$-robot, that is required to repeat a circular motion in the oper- ational space with frequency $\omega 0=7.0 \mathrm{rad}$ $\sec ^{-1}$, cen- ${ }^{2}{ }^{2}=x_{1}^{2} x_{2}^{1 / 2}$, radius $\rho=0.5$ and a step time increment of .6.t $=10^{-3} \mathrm{sec}$. The goal here is to position the end-effector of the $n R$-robot at a target location while minimizing the joint angle drift using the fitness function $f 1$ with $A=B=1$. The initial joint configuration is obtained using the OLGA with the fitness function $f 2$.

The average of the positional error for $n c$ cycles is given by the expression:

Fig. 2 Procedure for the

OLGA

ulation of strings, with dimension $n P=N$, is constructed at random and the search is then carried out among this population. Each chromosome is defined

through an array of $n V=n$ values, $q i, i=1, \ldots, n$, represented as floating-point numbers initialized in the range $[q \min , q \max ]$. For the generation $T$, the $i$ th chro- mosome of the population is represented

$$
\text { ,q })^{\text {as: }}(T, i)=\left(\begin{array}{ccc}
q^{(T} & \quad \text { (ii) }) \\
1 & \ldots, q_{n}
\end{array}\right)
$$

The end-effector position, $\mathbf{x}^{(T, i)}$, for each tion, $\mathbf{q}^{(T, i)}$, is easily calculated using the direct kinematics.

\subsubsection{Optimization criteria}

The evaluation function is defined based on the posi- tional error of the end-effector:

$$
\text { Perror } \overline{(x c-x f)^{2}+(y c-y f)^{2}}
$$

$=$

where $\mathbf{x}_{\mathrm{C}}=\left(x_{\mathrm{c}}, y_{\mathrm{c}}\right)$ and $\mathbf{x f}=\left(x \mathrm{f}, \mathrm{yf}_{\mathrm{f}}\right)$ are vectors representing the end-effector current position and the de-

$$
\begin{aligned}
& \bar{P} \text { error } \frac{\zeta_{i_{i=1}} \text { Perror }}{k} \\
& =
\end{aligned}
$$

where $k$ is the number of sampling points which is defined as:

$k=\frac{2 \pi}{\omega .6 . t} n C$

The average of the total joint displacement for the $n R$ -

robot is given by the expression:

$$
\overline{.6 . q}=\frac{\mathbf{l . 6 . q \mathrm { I }}}{n}
$$

where .6.q $=\mathbf{q f}-\mathbf{q 0}$ is the vector of the joint displacement between the final, $\mathbf{q} \mathbf{f}$, and the initial, q0, joint configurations, and $|\cdot|$ represents the Euclidean norm.

The CLGA algorithm adopts crossover probabilities of $p_{\mathrm{C}}=0.5$ for $n=\{3,4,5,6,7\}$ and mutation probabilities of $p_{\mathrm{m}}=0.5$ and $p_{\mathrm{m}}=0.3$ for $n=$ sired final position, respectively. Therefore, the algorithm minimizes the function $f 2=$ Perror. 


\section{Simulation results}

In this section we start by analyzing the performance of the CLGA for a free workspace and then we study the effect of including several types of obstacles in the working environment.
$\{3,4\}$ and $n=\{5,6,7\}$, respectively. The string population is $n P=\{200,400,800,1200,1600\}$ for $n=$ $\{3,4,5,6,7\}$, respectively, and the results are obtainedfor $n G=200$ consecutive generations. Each variable value is initialized in the range $[-1,1]$.

The OLGA algorithm adopts crossover and mutation probabilities of $p_{\mathrm{C}}=0.5$ and $p_{\mathrm{m}}=0.5$, respectively, a string population of $n P=1600$, and the results are obtained for $n G=200$ consecutive generations. Each variable value is initialized in the range $[-2 \pi, 2 \pi]$. 
4.1 The CLGA performance in a workspace without obstacles

The average of the positional error, $\bar{P}$ error, for $n$ $=$

$\{3,4,5,6,7\}$ rotational joints, $n C=50$ cycles and radial distance $r=\{0.7,1.0,2.0\}$, is depicted in Fig. 3 .

We observe that:

(i) the CLGA gives good precision in the task of positioning the end-effector at the target position;

(ii) in general, we get better results for the radial dis- tance $r=2.0$ and worse results for the radial dis- tance $r=0.7$; (iii) in general, the positional error gets worse when the number of joints increases.

Figures 4-5 show successive robot configurations, for $n=\{3,7\}$ rotational joints and $r=$ 2.0 , during the 1 st and 50th cycles, respectively. As we can see, the joint configurations are very similar, for both cycles,

revealing that the joint positions are repetitive. For comparison purposes, Figs. $6-7$ show successive robot configurations, for $n=\{3,7\}$ rotational joints and $r=2.0$, during the 1st and 50th cycles, respectively, for the CLP method. As we can see, the joint configurations are very different, revealing that the joint posi- tions are not repetitive.
Fig. $3 \bar{P}$ error of the $n R$-robot during $n C=$ 50 cycles for $n=\{3,4$, $5,6,7\}$ and $r=\{0.7,1.0,2.0\}$

Fig. 4 CLGA successive robot configurations of the $3 R$-robot for the 1 st and 50th cycles, respectively, and $r=2.0$
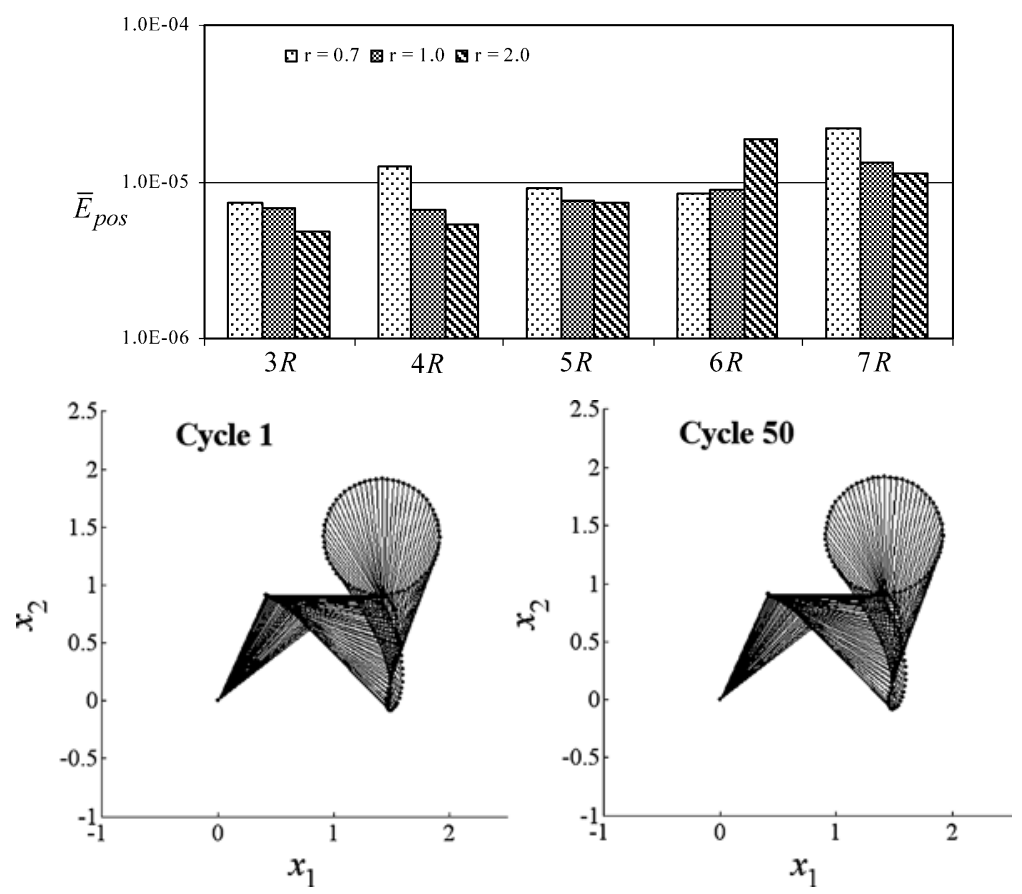
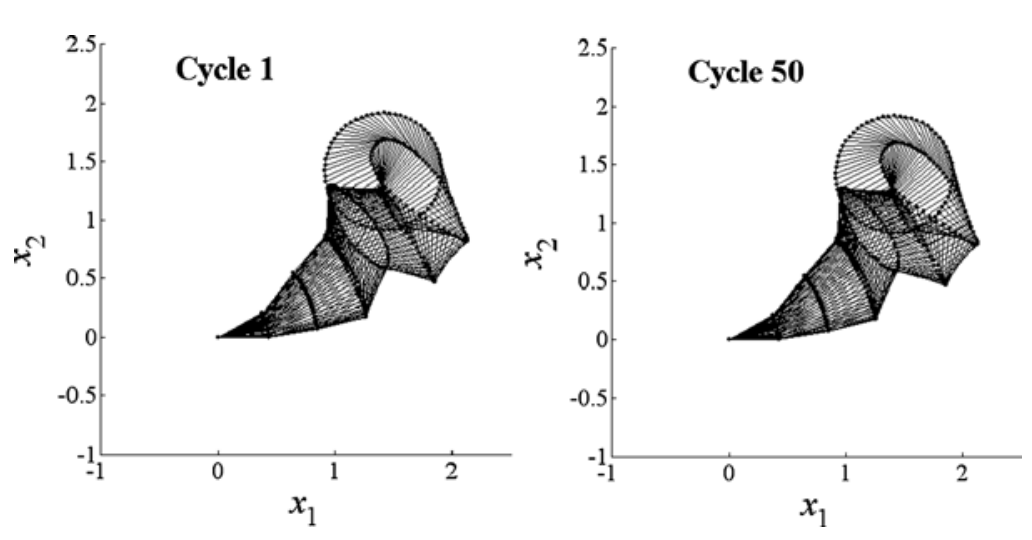

Fig. 5 CLGA successive robot configurations of the $7 R$-robot for the 1st and50th cycles, respectively, and $r=2.0$ 
Fig. 6 CLP successive robot configurations of the $3 R$-robot for the 1 st and 50 th cycles, respectively, and $r=2.0$
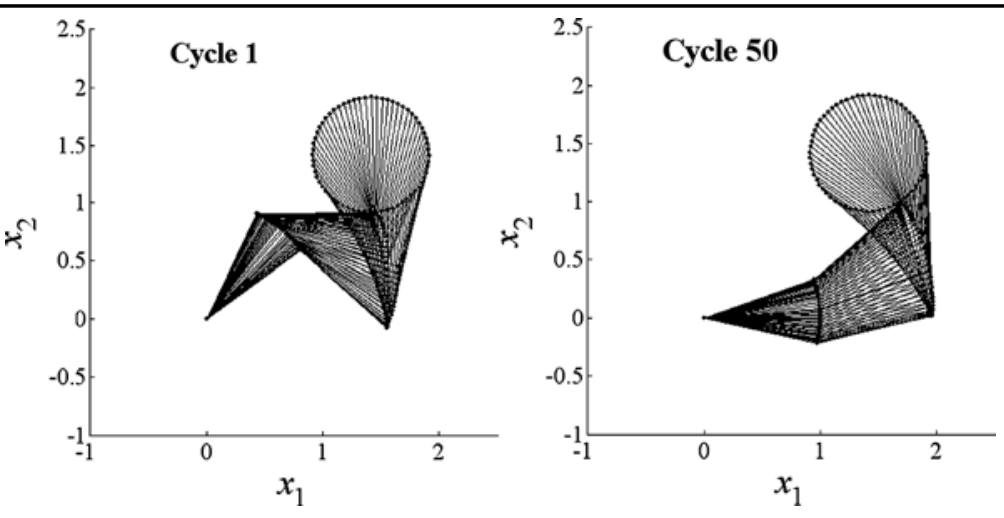

Fig. 7 CLP successive robot configurations of the $7 R$-robot for the 1st and50th cycles, respectively, and $r=2.0$
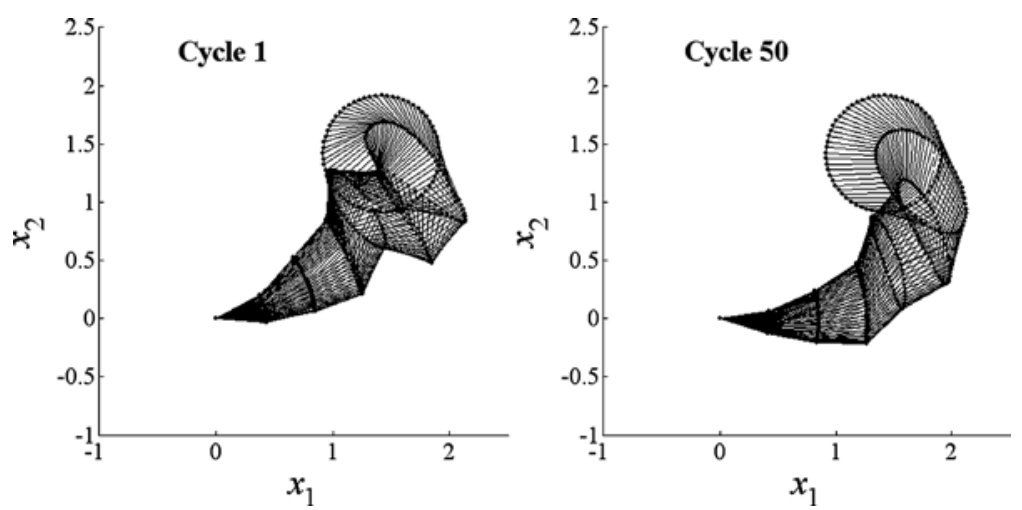

Table 1 CLGA and CLP average of the total joint displacement, .6.q, of the $n R$-robot, $r=\{0.7,1.0,2.0\}$ and after $n C$ $=50$ cycles

\begin{tabular}{llllllll}
\hline CLGA & $r=0.7$ & $r=1.0$ & $r=2.0$ & CLP & $r=0.7$ & $r=1.0$ & $r=2.0$ \\
\hline $3 R$ & $9.96 \mathrm{E}-04$ & $8.84 \mathrm{E}-04$ & $1.08 \mathrm{E}-03$ & $3 R$ & $1.35 \mathrm{E}+01$ & $6.41 \mathrm{E}+00$ & $5.80 \mathrm{E}-01$ \\
$4 R$ & $7.12 \mathrm{E}-04$ & $7.38 \mathrm{E}-04$ & $5.70 \mathrm{E}-04$ & $4 R$ & $8.2 \mathrm{E}+00$ & $4.4 \mathrm{E}+00$ & $5.8 \mathrm{E}-01$ \\
$5 R$ & $6.73 \mathrm{E}-04$ & $5.42 \mathrm{E}-04$ & $6.15 \mathrm{E}-04$ & $5 R$ & $7.2 \mathrm{E}+00$ & $2.2 \mathrm{E}+00$ & $4.4 \mathrm{E}-01$ \\
$6 R$ & $5.98 \mathrm{E}-04$ & $4.81 \mathrm{E}-04$ & $8.57 \mathrm{E}-04$ & $6 R$ & $5.4 \mathrm{E}+00$ & $4.9 \mathrm{E}+00$ & $3.0 \mathrm{E}-01$ \\
$7 R$ & $1.26 \mathrm{E}-03$ & $5.44 \mathrm{E}-04$ & $5.39 \mathrm{E}-04$ & $7 R$ & $4.2 \mathrm{E}+00$ & $2.4 \mathrm{E}+00$ & $2.0 \mathrm{E}-01$ \\
\hline
\end{tabular}

In order to evaluate the joint angle drift after ex- ecuting $n c=50$ cycles, Table 1 shows the average of the total joint displacement, .6.q, when $n$ $=$

$\{3,4,5,6,7\}$ and $r=\{0.7,1.0,2.0\}$, for the CLGA and the CLP methods. We verify that for the CLGA we get some drift in joint positions, but the values are very similar for all the manipulators and for all of the radial distances. For the CLP we get a high drift in the joint positions revealing that this method leads to unpredictable arm configurations.

Figures 8-9 show the joint positions and the Fourier transform of the robot joint velocities for the 1st joint and $n C=50, n=\{3,7\}$ and $r=\{0.7,2.0\}$, with the 
CLGA and the CLP methods. For the other joints the results are similar to those of the 1st joint.

For the CLGA, we conclude that:

(i) repetitive trajectories in the operational space lead to periodic trajectories in the joint space;

(ii) the initial and final joint positions, for each of the cycles, are very close;

(iii) the signal energy is concentrated essentially in the fundamental and multiple higher harmonics.

For the CLP, we conclude that the results depend on the circle being executed. Besides the position drifts, unpredictable motions with severe variations 


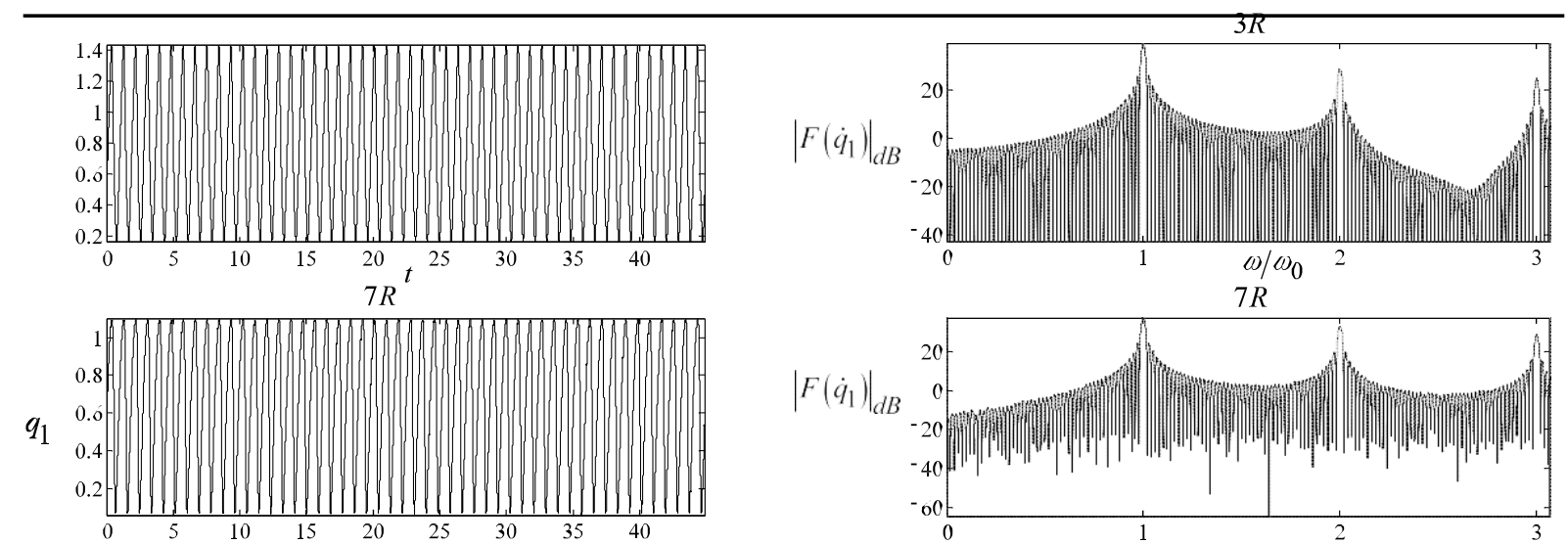

$t$

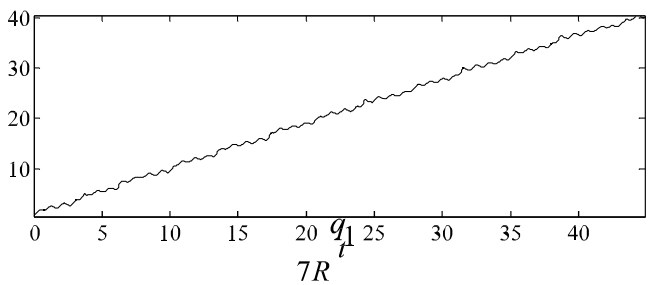

(a)

$\left|F\left(\dot{q}_{1}\right)\right|_{d B}$
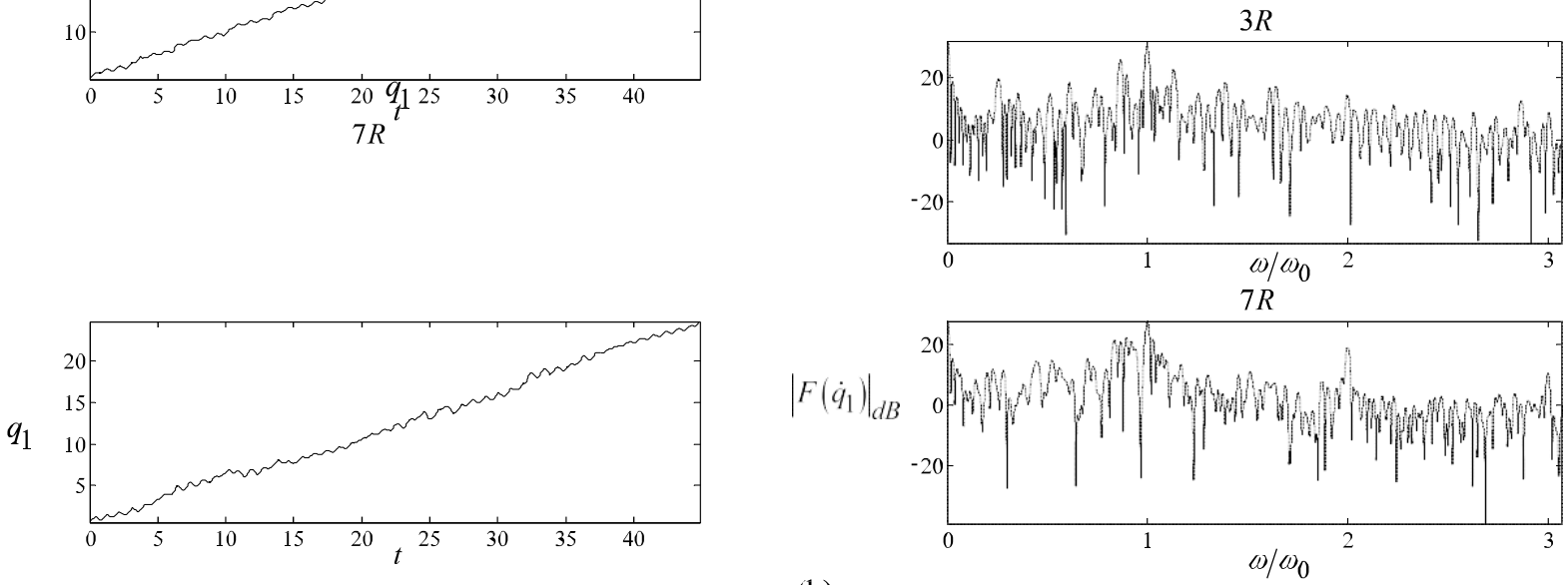

(b)

Fig. 8 The $\{3,7\} R$-robot 1st joint positions vs. time and $|F\{\dot{q}(t)\}|$ vs. $\omega / \omega 0$ during $n C=50$ cycles for $r=0.7$ and (a) CLGA, (b) CLP

occur that lead to high joint transients. Moreover, for $r=0.7$ we verify that a large part of the energy is distributed along several sub-harmonics, revealing

properties similar to those that occur in chaotic systems.

We verify that the CLGA has a good performance in repetitive motion tasks because we get not only a good positioning but also a repetitive behavior.

\subsection{The CLGA performance in a workspace with} obstacles
This section presents the results of several simulations, when considering two obstacles in the workspace. At the end of the section, some examples in a workspace 
with three obstacles are presented. When, for a given joint configuration, some part of the manipulator is inside an obstacle, the CLGA simply rejects the configuration and generates a new population ele- ment.

\subsubsection{The CLGA performance in a workspace with two obstacles}

In the experiments, the position of the obstacles in the workspace depends on the radial distance. Fig- ures 10-12 represent the positions of the obstacles in the workspace for each radial distance, $O(r ; i)$, for $r=\{0.7,1.0,2.0\}$ and $i=1,2,3$.

The average of the positional error, $P$ error, for $n$

$$
=
$$

$\{3,4,5,6,7\}, n C=50$ cycles and $O(r ; i)$ for $r=$ 

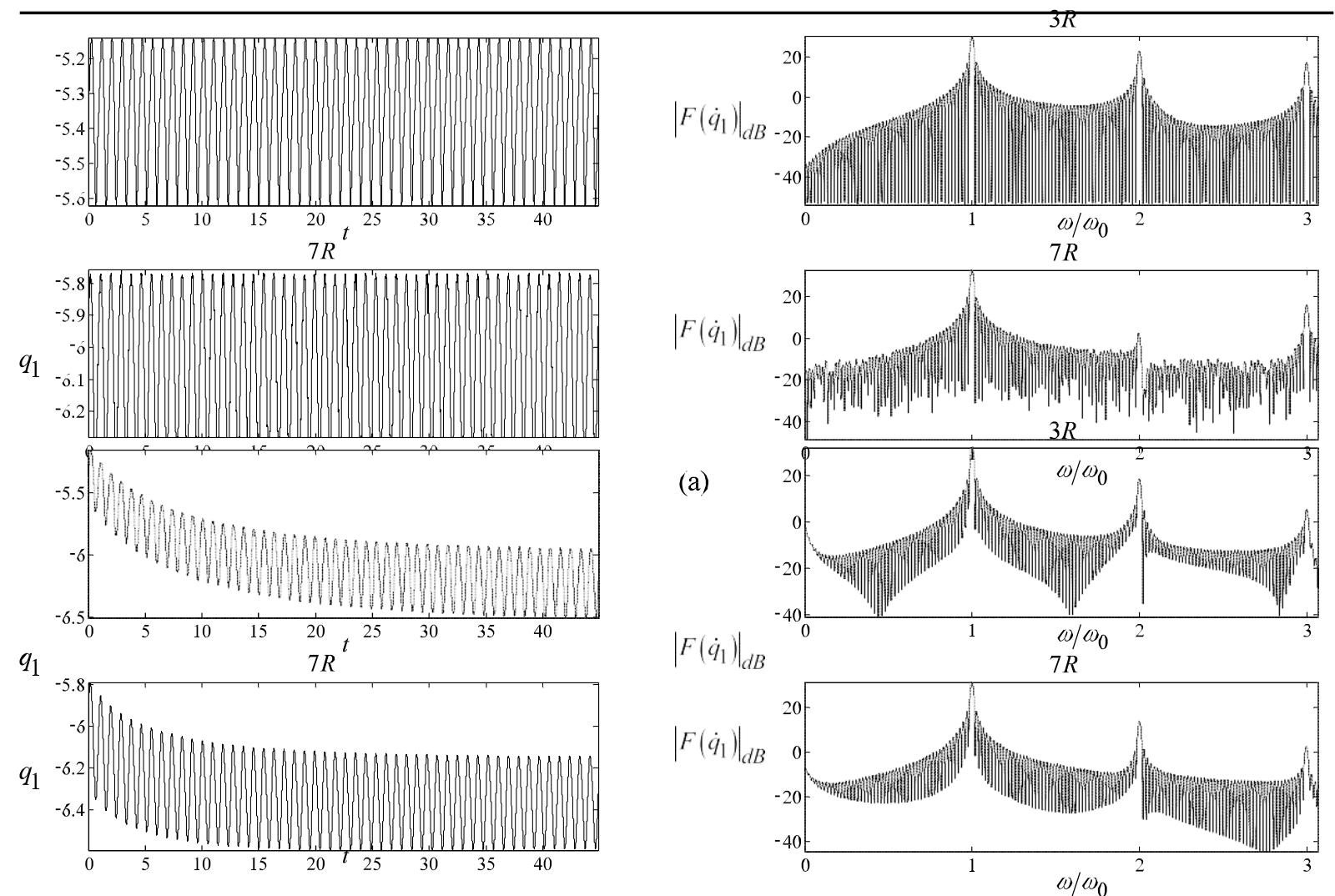

(b)

Fig. 9 The $\{3,7\} R$-robot 1 st joint positions vs. time and $|F\{\dot{q}(t)\}|$ vs. $\omega / \omega 0$ during $n C=50$ cycles for $r=2.0$ and (a) CLGA, (b) CLP
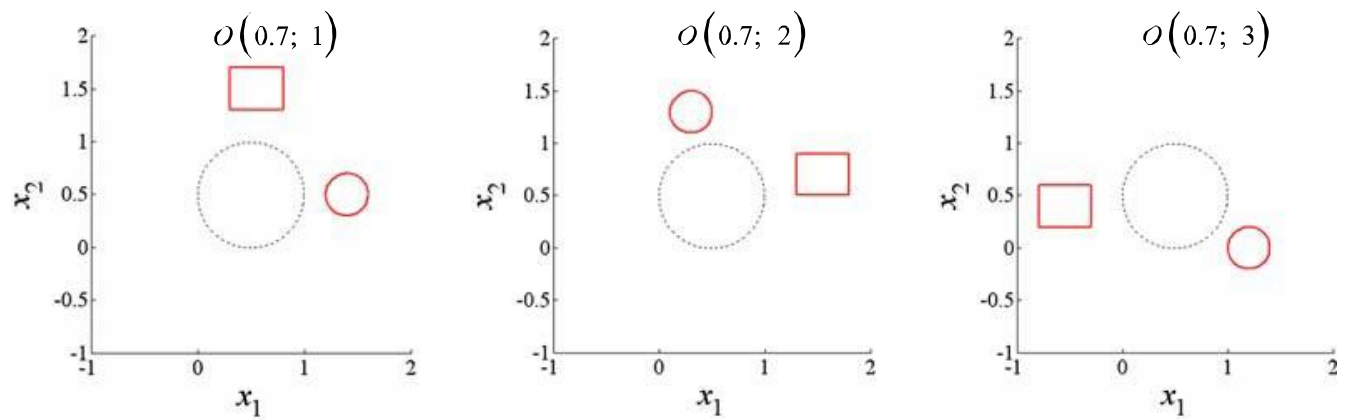

Fig. 10 Workspaces for $O(0.7 ; i), i=1,2,3$

$\{0.7,1.0,2.0\}, i=1,2,3$, is presented in Figs. 13-15, respectively.

We observe that: (i) the CLGA gives good precision in the task of positioning the end-effector at the target position, while avoiding the obstacles in the workspace; 

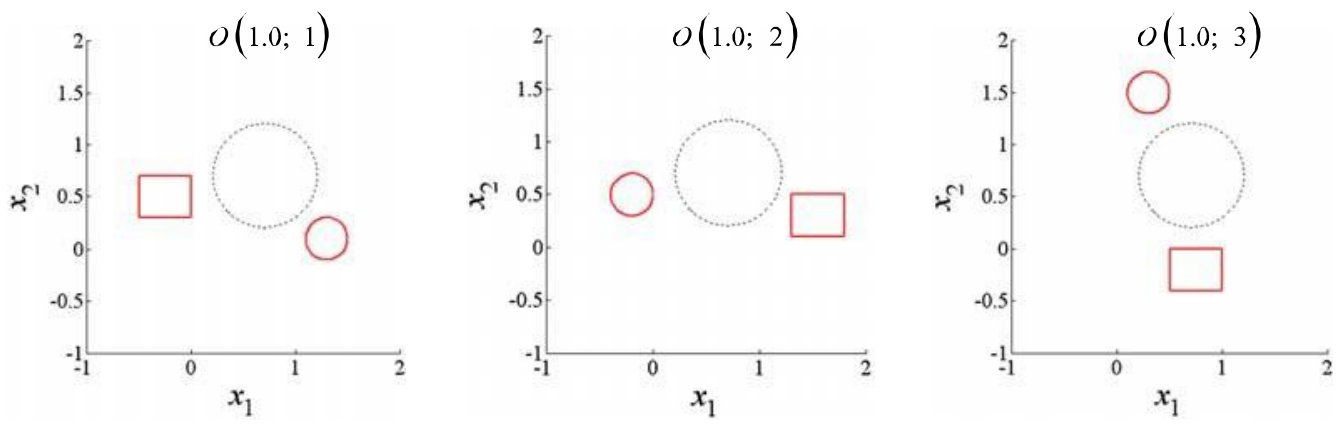

Fig. 11 Workspaces for $O(1.0 ; i), i=1,2,3$
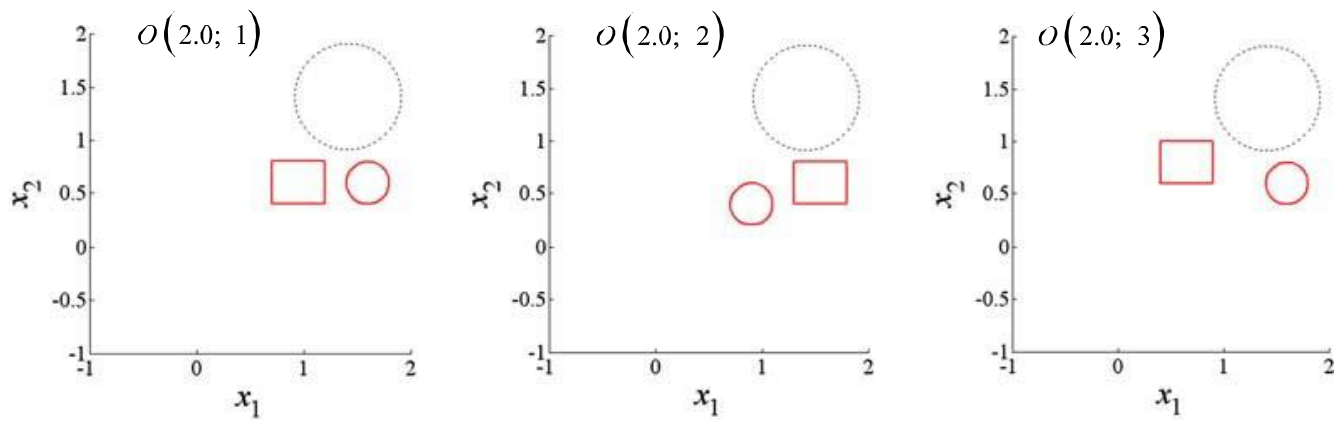

Fig. 12 Workspaces for $O(2.0 ; i), i=1,2,3$

Fig. $13 \bar{P}$ error of the $n R$-robot during $n C=50$ cycles for $O(0.7 ; i)$, $i=1,2,3$

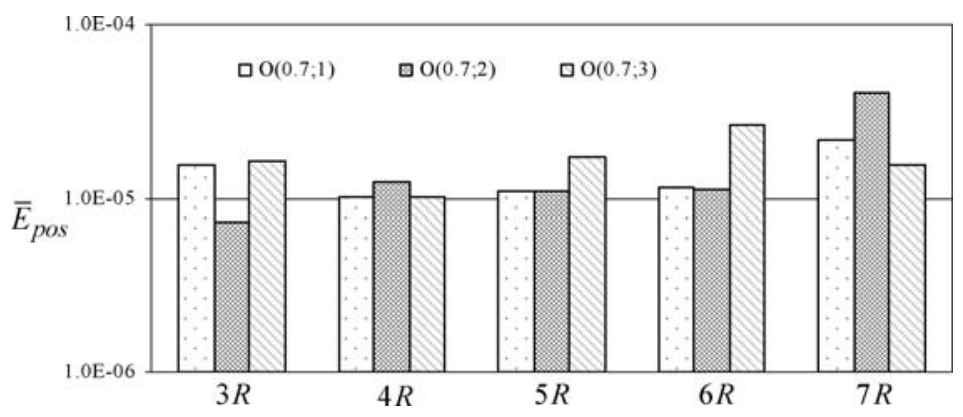

Fig. $14 \bar{P}$ error of the $n R$-robot during $n C=50$ cycles for $O(1.0 ; i)$, $i=1,2,3$

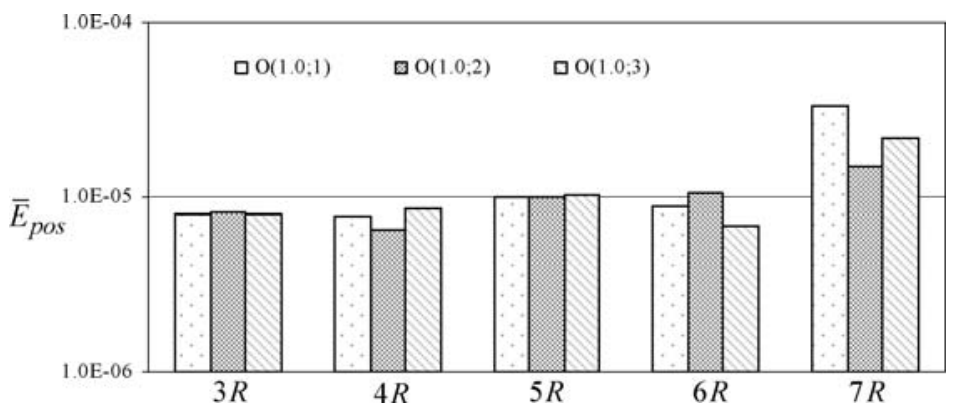


Fig. $15 P_{\text {error }}$ of the $n R$-robot during $n_{C}=50$ cycles for $O(2.0 ; i)$, $i=1,2,3$
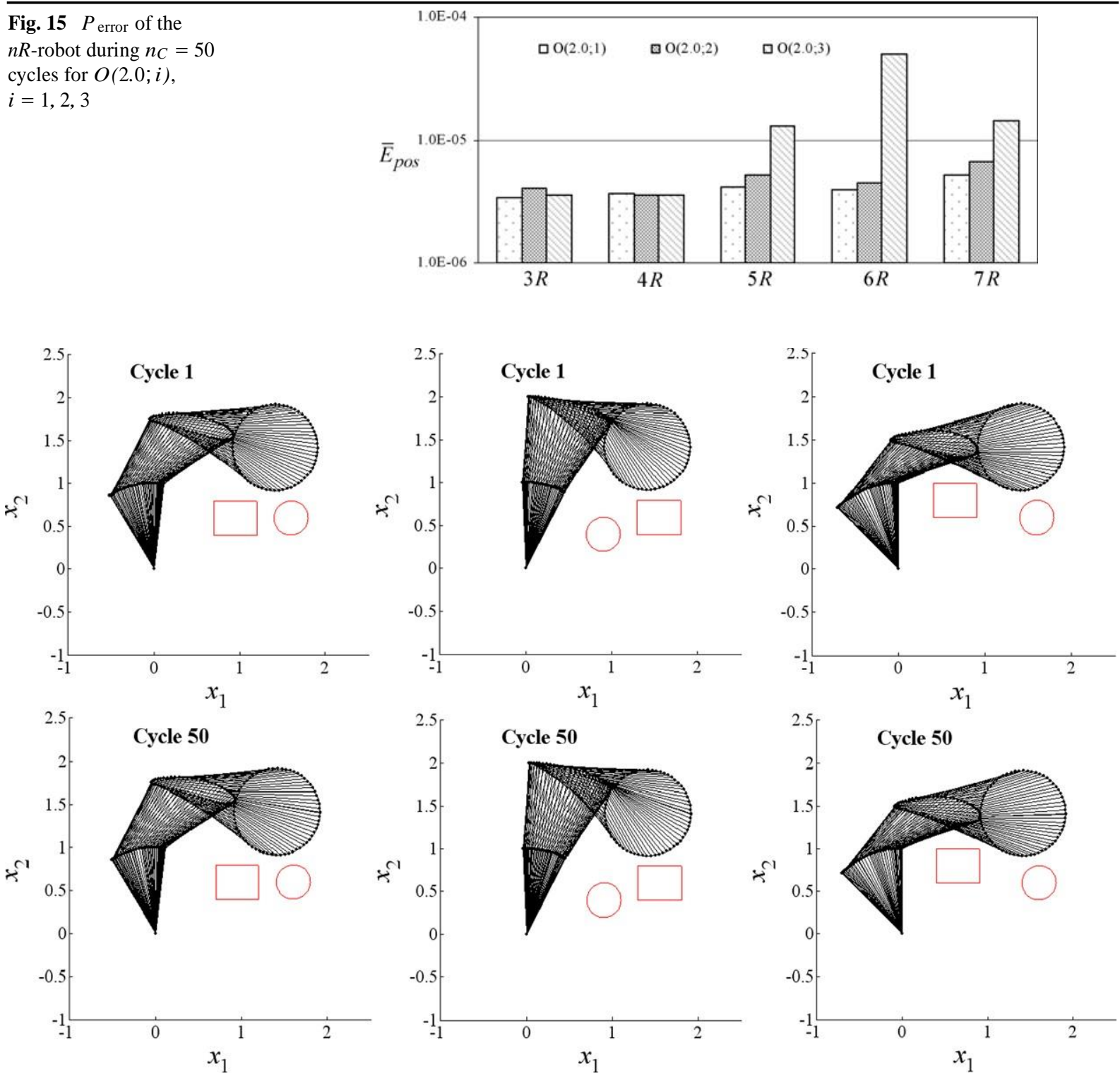

Fig. 16 Successive robot configurations of the $3 R$-robot for the 1 st and 50th cycles, respectively, and $O(2.0 ; i), i=$ $1,2,3$

(ii) in general, we get better results for the radial dis- tance $r=2.0$ and worse results for $r=0.7$;

(iii) in general, the positional error, $P$ error, gets worse when the number of joints increases.

Figures 16-17 show successive robot configurations, for $n=\{3,7\}$ and $O(2.0 ; i), i=1,2,3$, during the 1st and 50th cycles, respectively, revealing that the motion is repetitive.

Table 2 shows the average of the joint displace- ment, .6.q, for $n=\{3,4,5,6,7\}$, and $O(r ; i), r=$ $\{0.7,1.0,2.0\}, i=1,2,3$. We conclude that the results 
are consistent with those we obtained in a workspace without obstacles.

Figures 18-20 show the joint positions and the Fourier transform of the robot joint velocities for the 1st joint and $n C=50$ cycles, $n=\{3,7\}$, $O(2.0 ; i)$,

$i=1,2,3$. For the others joints the results are similar to the verified ones for the 1 st joint.

We conclude that:

(i) repetitive trajectories in the operational space lead to periodic trajectories in the joint space; 

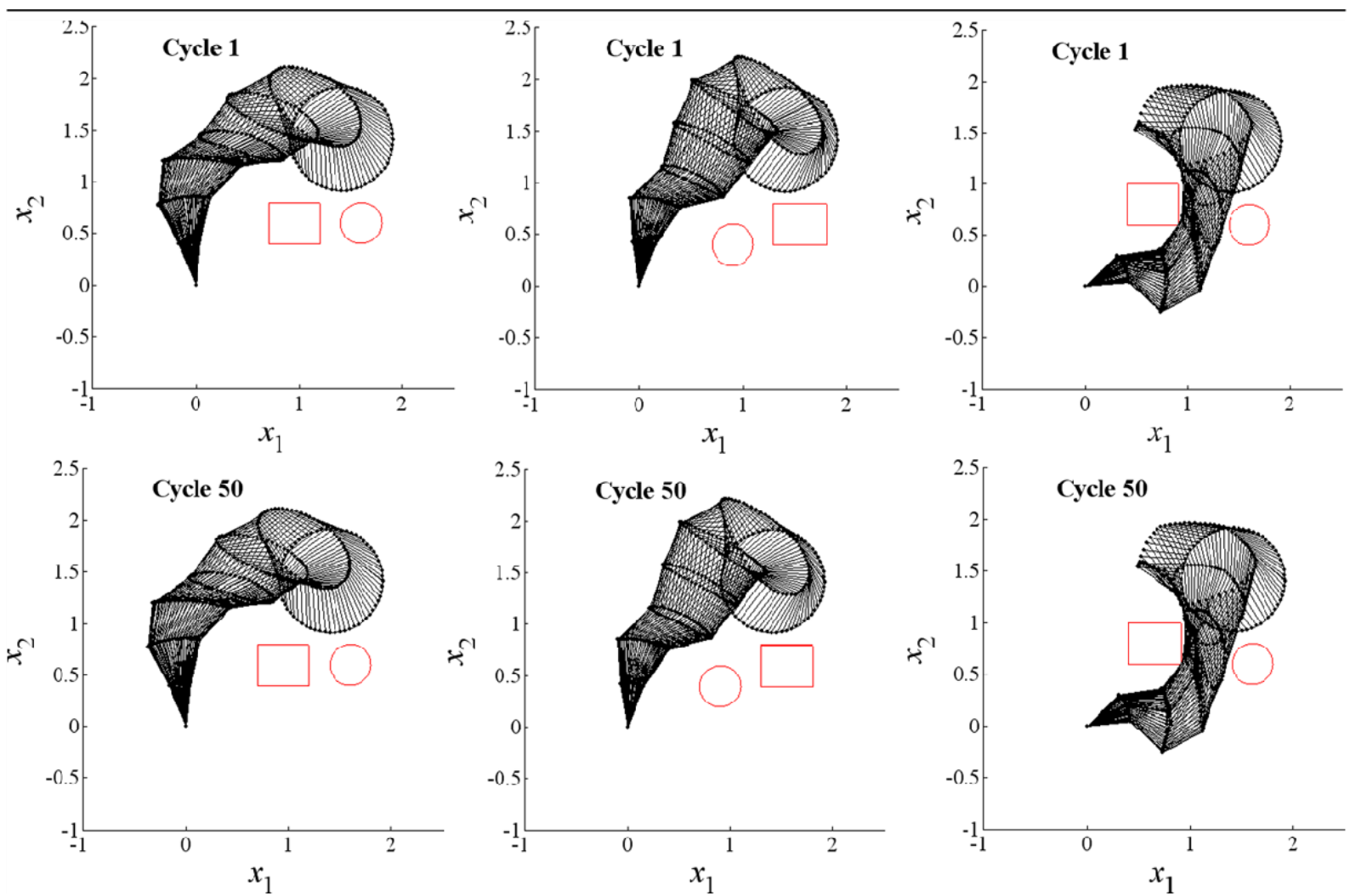

Fig. 17 Successive robot configurations of the $7 R$-robot for the 1 st and 50th cycles, respectively, and $O(2.0 ; i), i=$ $1,2,3$

Table 2 The average of the joint displacement of the $\overline{n R}$-robot, .6.q, after $n C=50$ cycles for $O(r ; i), r=\{0.7,1.0$, $2.0\}, i=1,2,3$

\begin{tabular}{llllllllll}
\hline & $O(0.7 ; 1)$ & $O(0.7 ; 2)$ & $O(0.7 ; 3)$ & $O(1.0 ; 1)$ & $O(1.0 ; 2)$ & $O(1.0 ; 3)$ & $O(2.0 ; 1)$ & $O(2.0 ; 2)$ & $O(2.0 ; 3)$ \\
\hline $3 R$ & $8.63 \mathrm{E}-04$ & $1.11 \mathrm{E}-03$ & $1.02 \mathrm{E}-03$ & $7.57 \mathrm{E}-04$ & $7.63 \mathrm{E}-04$ & $8.02 \mathrm{E}-04$ & $6.15 \mathrm{E}-04$ & $6.64 \mathrm{E}-04$ & $6.51 \mathrm{E}-04$ \\
$4 R$ & $6.16 \mathrm{E}-04$ & $6.86 \mathrm{E}-04$ & $5.98 \mathrm{E}-04$ & $6.07 \mathrm{E}-04$ & $7.39 \mathrm{E}-04$ & $6.85 \mathrm{E}-04$ & $4.29 \mathrm{E}-04$ & $4.19 \mathrm{E}-04$ & $4.22 \mathrm{E}-04$ \\
$5 R$ & $7.31 \mathrm{E}-04$ & $7.04 \mathrm{E}-04$ & $5.40 \mathrm{E}-04$ & $5.63 \mathrm{E}-04$ & $4.63 \mathrm{E}-04$ & $4.74 \mathrm{E}-04$ & $3.56 \mathrm{E}-04$ & $4.16 \mathrm{E}-04$ & $7.14 \mathrm{E}-04$ \\
$6 R$ & $4.66 \mathrm{E}-04$ & $6.28 \mathrm{E}-04$ & $5.92 \mathrm{E}-04$ & $4.67 \mathrm{E}-04$ & $3.45 \mathrm{E}-04$ & $6.23 \mathrm{E}-04$ & $2.38 \mathrm{E}-04$ & $2.96 \mathrm{E}-04$ & $7.33 \mathrm{E}-04$ \\
$1 R$ & $\underline{5.16 \mathrm{E}-04}$ & $3.75 \mathrm{E}-04$ & $3.95 \mathrm{E}-04$ & $4.63 \mathrm{E}-04$ & $5.39 \mathrm{E}-04$ & $2.95 \mathrm{E}-04$ & $7.07 \mathrm{E}-04$ & $3.94 \mathrm{E}-04$ & $1.96 \mathrm{E}-03$ \\
\hline
\end{tabular}

(ii) the initial and final joint positions for each one of the cycles are very close;

(iii) the signal energy is concentrated essentially in the fundamental and multiple higher harmonics.

We observe also that the results are consistent with those of the previous section and that the presence of obstacles does not present an additional complexity for the CLGA to reach a repetitive solution.

\subsubsection{The CLGA performance in a workspace with three obstacles}

One of the problems that occur when we use the repeatability criterion is that, if the initial joint configu- 
ration of the manipulator is not well adjusted for the task, then 'jumps' of the robot structure will occur to prevent the collision with the obstacles. In the experi- ments the trajectory is repetitive and, therefore, these jumps may occur during several cycles.

In Figs. 21-22 we use a workspace with three ob- stacles. For $n=3$, after two different executions of the algorithm, we obtained two different initial joint con-

figurations with distinct consequences, in terms of the positional error and performance in the execution of the task. However, if we use a manipulator with $n=7$ rotational joints, there is no problem to find a good solution for the initial positions. Anyway, the manip- 
$3 R$
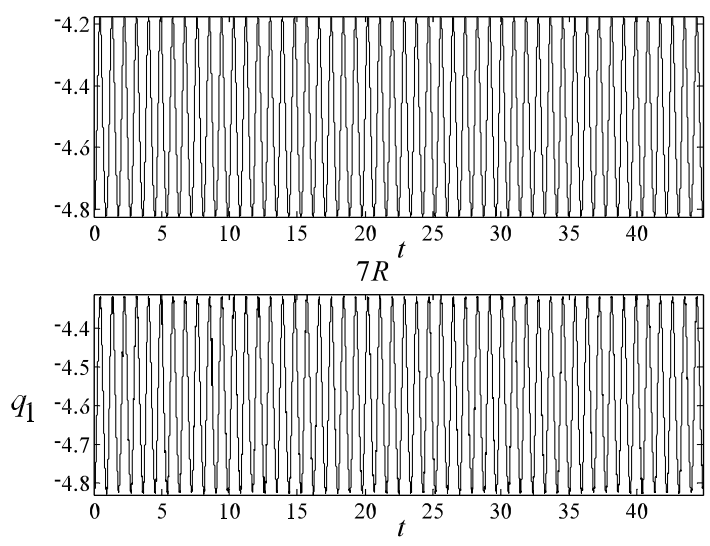
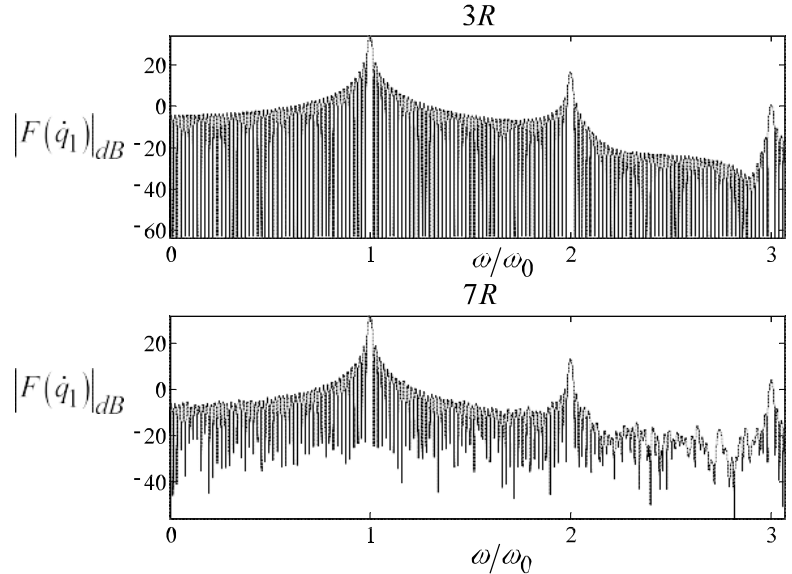

Fig. 18 The $\{3,7\} R$-robot 1 st joint positions vs. time and $|F\{\dot{q}(t)\}|$ vs. $\omega / \omega 0$ during $n C=50$ cycles for $O(2.0 ; 1)$
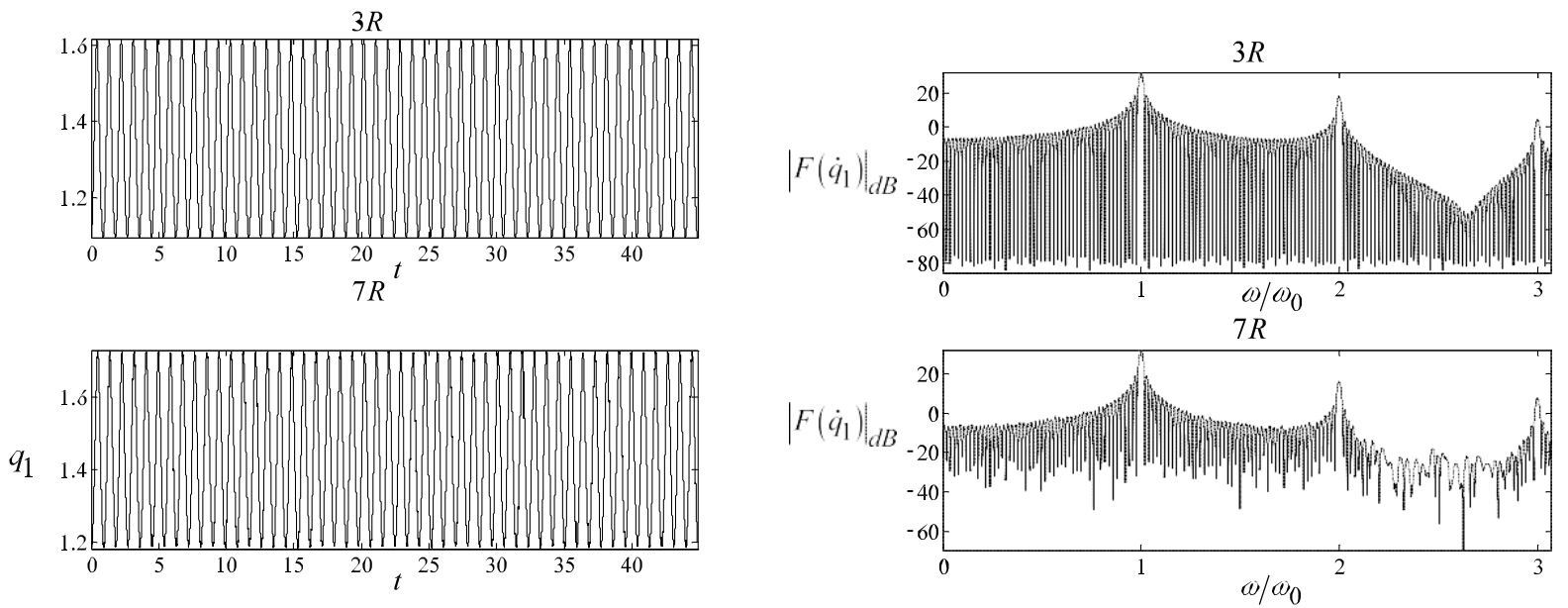

Fig. 19 The $\{3,7\} R$-robot 1 st joint positions vs. time and $|F\{\dot{q}(t)\}|$ vs. $\omega / \omega 0$ during $n C=50$ cycles for $O(2.0 ; 2)$

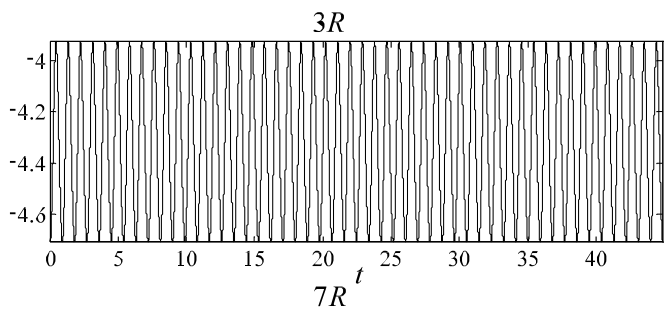



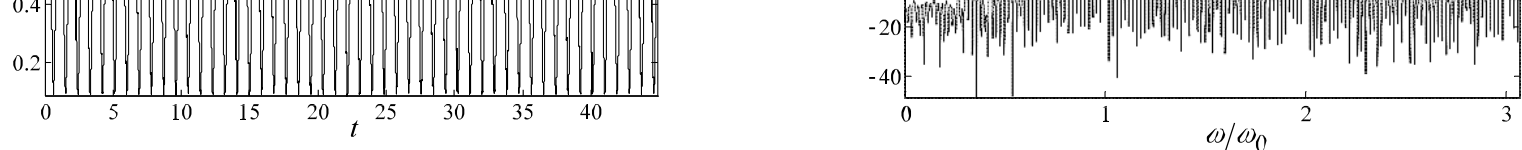

Fig. 20 The $\{3,7\} R$-robot 1 st joint positions vs. time and $|F\{\dot{q}(t)\}|$ vs. $\omega / \omega 0$ during $n C=50$ cycles for $O(2.0 ; 3)$ 
Fig. 21 Successive robot configurations of the $3 R$-robot for the 1 st and 2nd cycles, respectively, for a workspace with three obstacles and $r=2.0$, with two different initial joint configurations
Fig. 22 Successive robot configurations of the $7 R$-robot for the 1 st and2nd cycles, respectively, for a workspace with three obstacles and $r=2.0$
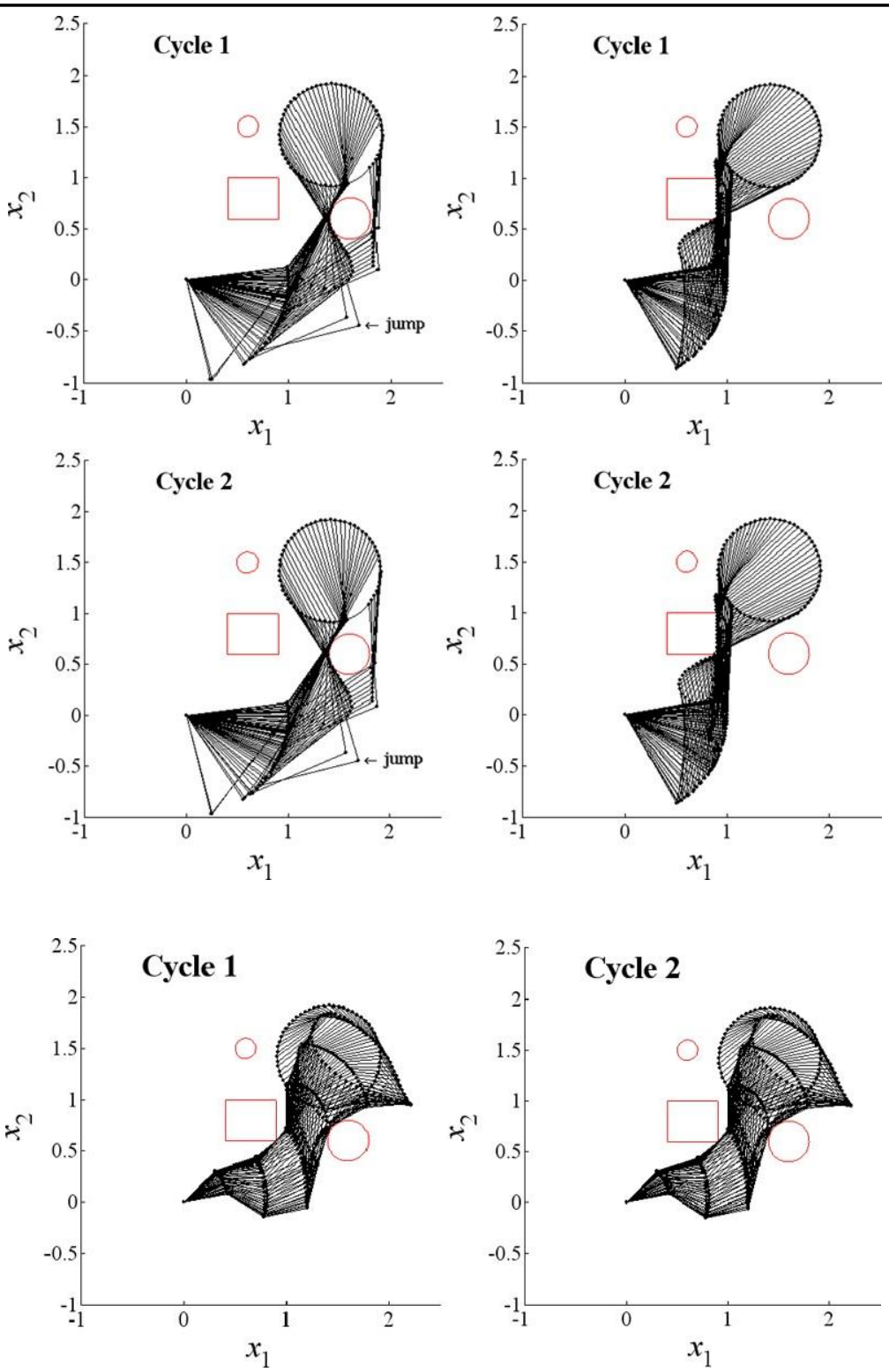

ulator motion is always repeatable and the drift in the joint positions is similar to the one we obtained in the previous experiments.

\section{Conclusions}

A CLGA algorithm that combines the CLP with a GA scheme was presented. Several experiments were de- veloped to study the performance of the CLGA when the manipulator is required to repeat a circular motion in the operational space, while satisfying an optimiza- 
tion criterion, in a workspace without and with obsta- cles.

The results show that the CLGA gives good results in the perspective of the positional error and the re- peatability of the joint positions. In what concerns the drift in the joint angles, there is no zero-drift but the re- sults seem to be acceptable for all manipulators under analysis.

It is shown that the presence of obstacles does not present an additional complexity for the CLGA. How- ever, the initial configuration of the manipulator plays an important role in the performance of the manipu- 
lator in a workspace with obstacles. In fact, if the ini- tial configuration is not adjusted adequately for the re- quired task, then jumps may occur in the joint posi- tions to prevent the collision with the obstacles.

\section{References}

1. Nenchev, D.N., Tsumaki, Y.: Motion analysis of a kine- matically redundant seven-DOF manipulator under the singularity-consistent method. In: Proc. of the 2003 IEEE Int. Conf. on Robotics and Automation, pp. 2760-2765 (2003)

2. Doty, K.L., Melchiorri, C., Bonivento, C.: A theory of gen- eralized inverses applied to robotics. Int. J. Robotics Res. 12, 1-19 (1993)

3. Baillieul, J., Hollerbach, J., Brockett, R.: Programming and control of kinematically redundant manipulators. In: Proc. of the 23rd IEEE Conf. on Decision and Control, pp. 768- 774 (1984)

4. Klein, C.A., Huang, C.-H.: Review of pseudoinverse con- trol for use with kinematically redundant manipulators. IEEE Trans. Syst. Man Cybern. 13(3), 245-250 (1983)

5. Roberts, R.G., Maciejewski, A.: Repeatable generalized in- verse control strategies for kinematically redundant ma- nipulators. IEEE Trans. Autom. Control 38(5), 689-699 (1993)

6. Klein, C.A., Ahmed, S.: Repeatable pseudoinverse con- trol for planar kinematically redundant manipulators. IEEE Trans. Syst. Man Cybern. 25(12), 1657-1662 (1995)

7. Zhang, Y., Zhu, H., Lv, X., Li, K.: Joint angle drift problem of PUMA560 robot arm solved by a simplified LVI-based primal-dual neural network. In: Proc. of the IEEE Int. Conf. on Industrial Technology, pp. 1-26 (2008)

8. Zhang, Y., Lv, X., Li, Z., Yang, Z., Zhu, H.: Effective neural remedy for drift phenomenon of planar three-link robot arm using quadratic performance index. Electron. Lett. 44(6), 436437 (2008). doi:10.1049/el:20080455

9. Zhang, Y., Tan, Z., Yang, Z., Lv, X.: A dual neural network applied to drift-free resolution of fivelink planar robot arm. In: Proc. of the 2008 IEEE International Conference on In- formation and Automation, pp. 1274-1279 (2008)

10. Baillieul, J.: Kinematic programming alternatives Int. Conf. on Robotics and Automation, pp. 722728 (1985)

11. Baker, D.R., Wampler II, C.W.: On the inverse kinematics of redundant manipulators. Int. J. Robotics Res. 7(211), 3- 21 (1988)

12. Park, K.-C., Chang, P.-H., Lee, S.: A new kind of singular- ity in redundant manipulation: semialgorithmic singular- ity. In: Proc. of the IEEE Int. Conf. on Robotics and Au- tomation, vol. 2, pp. 1979-1984 (2002)

13. Park, J., Chung, W.-K., Youm, Y.: Characteristics of op- timal solutions in kinematics resolutions of redundancy. IEEE Trans. Robotics Autom. 12(3), 471-478(1996) 
14. Goldenberg, D.E.: Genetic Algorithms in Search Opti- mization, and Machine Learning. AddisonWesley, Read- ing (1989)

15. Parker, J.K., Khoogar, A.R., Goldberg, D.E.: Inverse kine- matics of redundant robots using genetic algorithms. In: Proc. of the 1989 IEEE Int. Conf. on Robotics and Automa- tion, pp. 271-276 (1989)

16. Arakawa, T., Kubota, N., Fukuda, T.: Virusevolutionary genetic algorithm with subpopulations: application to tra- jectory generation of redundant manipulator through en- ergy optimization. In: Proc. of the 1996 IEEE Int. Conf. on Systems, Man, and Cybernetics, pp. 14-17(1996)

17. Kubota, N., Arakawa, T., Fukuda, T., Shimojima, K.: Tra- jectory generation for redundant manipulator using virus evolutionary genetic algorithm. In: Proc. of the IEEE Int. Conf. on Robotics and Automation, pp. 205-210 (1997)

18. de la Cueva, V., Ramos, F.: Cooperative genetic algorithms: a new approach to solve the path planning problem for cooperative robotic manipulators sharing the same work space. In: Proc. of the IEEE/RSJ Int. Conference on Intelligent Robots and Systems, pp. 267-272 (1998)

19. Nishimura, T., Sugawara, K., Yoshihara, I., Abe, K.: A mo- tion planning method for a hyper multi-joint manipulator using genetic algorithm. In: Proc. of the IEEE Int. Conf. on Systems, Man, and Cybernetics, pp. 645-650 (1999)

20. McAvoy, B., Sangolola, B.: Optimal trajectory generation for redundant planar manipulators. In: Proc. of the IEEE Int. Conf. on Systems, Man, and Cybernetics, pp. 3241-3246 (2000)

21. Peng, Y., Wei, W.: A new trajectory planning method of re- dundant manipulator based on adaptive simulated anneal- ing genetic algorithm (ASAGA). In: Proc. of the IEEE Int. Conf. on Computational Intelligence and Security, pp. 262- 265 (2006)

22. Zhang, Y., Sun, Z., Yang, T.: Optimal motion generation of a flexible macro-micro manipulator system using genetic algorithm and neural network. In: Proc. of the 2006 IEEE Conf. on Robotics, Automation and Mechatronics, pp. 1-6 (2006)

23. Pires, E.J.S., Machado, J.A.T., Oliveira, P.B.M.:
Manipula- tor trajectory planning using a MOEA. Appl. Soft Comput. J. 7(3), 659-667 (2007)

24. Whitney, D.E.: Resolved motion rate control of manipula- tors and human prostheses. IEEE Trans. Man Mach. Syst. 10(2), 47-53 (1969)

25. Siciliano, B.: Kinematic control of redundant robot manip- ulators: a tutorial. J. Intell. Robotic Syst. 3, 201-212 (1990)

26. Marcos, M.G., Duarte, F.B., Machado, J.A.T.: Complex dy- namics in the trajectory control of redundant manipulators. Trans. Nonlinear Sci. Complex. (World Scientific) 1, 134- 143 (2007)

27. Holland, J.H.: Adaptation in Natural and Artificial Systems. University of Michigan Press, Ann Arbor (1992) (2nd edn.: MIT Press, Cambridge) 